\title{
Processamento auditivo em teste e reteste: confiabilidade da avaliação
}

\author{
Test and retest of auditory processing: reliability of the \\ evaluation
}

\author{
Maria Fernanda Simões dos Santos Frascá ${ }^{1}$, Ivone Ferreira Neves Lobo ${ }^{2}$, Eliane Schochat ${ }^{3}$
}

\begin{abstract}
RESUMO
Objetivos: Verificar a confiabilidade de alguns dos testes que avaliam o Processamento Auditivo Central (PAC), por meio de um estudo do tipo teste-reteste. Métodos: Quarenta indivíduos voluntários, falantes do Português, foram sorteados ao acaso e avaliados quanto ao PAC nas situações de teste e reteste, com intervalos que variaram entre uma semana a um mês. Foram aplicados os testes de localização, memória sequencial verbal e não verbal, dois testes monóticos e dois dicóticos. Estes últimos, escolhidos de acordo com a faixa etária e condições de resposta de cada sujeito. Resultados: Não houve diferença entre as orelhas testadas, nem entre as situações de teste e reteste, de todo o grupo. Quando comparados os desempenhos dos indivíduos, cujos resultados apontaram para um transtorno do PAC, com aqueles cujos resultados estiveram dentro da normalidade, em ambas as situações houve variação na maior parte dos testes aplicados. Conclusão: Os testes do PAC utilizados neste estudo demonstraram sua confiabilidade por meio do teste-reteste.
\end{abstract}

Descritores: Audição; Transtornos da audição; Percepção auditiva; Transtornos da percepção auditiva; Testes auditivos

\section{INTRODUÇÃO}

A avaliação comportamental do Processamento Auditivo Central (PAC), embora recente no Brasil, é uma prática clínica bastante frequente devido à sua grande contribuição no diagnóstico audiológico.

O PAC foi definido como "o conjunto de mecanismos e processos do sistema nervoso auditivo responsável pelos fenômenos de localização, discriminação, reconhecimento, aspectos temporais da audição, incluindo: resolução temporal, mascaramento temporal, ordenação temporal, desempenho auditivo com mensagem competitiva e com sinais acústicos distorcidos"(1).

Os transtornos de PAC foram claramente definidos como

Trabalho realizado no Departamento de Fisioterapia, Fonoaudiologia e Terapia Ocupacional da Faculdade de Medicina, Universidade de São Paulo - USP - São Paulo (SP), Brasil.

(1) Departamento de Fisioterapia, Fonoaudiologia e Terapia Ocupacional da Faculdade de Medicina, Universidade de São Paulo - USP - São Paulo (SP), Brasil.

(2) Departamento de Fisioterapia, Fonoaudiologia e Terapia Ocupacional da Faculdade de Medicina, Universidade de São Paulo - USP - São Paulo (SP), Brasil.

(3) Curso de Fonoaudiologia do Departamento de Fisioterapia, Fonoaudiologia e Terapia Ocupacional da Faculdade de Medicina, Universidade de São Paulo - USP - São Paulo (SP), Brasil.

Endereço para correspondência: Eliane Schochat. Rua Cipotânea, 51, Butantã, São Paulo (SP), Brasil, CEP: 05360-160. E-mail: eschocha@usp.br Recebido em: 21/5/2010; Aceito em: 19/7/2010 uma alteração no processamento específico da modalidade auditiva, podendo estar associado a dificuldades em ouvir ou entender a fala, ao desenvolvimento da linguagem e à aprendizagem $^{(1)}$.

Os primeiros testes para a avaliação do sistema nervoso auditivo central surgiram na década de 1950 , tendo como principal finalidade a verificação da integridade da via auditiva $^{(2)}$. No entanto, a aplicação de testes comportamentais na avaliação da função auditiva em indivíduos com limiares auditivos normais surgiu na década de 1970, quando o conceito de PAC foi introduzido. Desde então, diversos testes vêm sendo desenvolvidos para a avaliação específica do PAC.

No Brasil, os testes comportamentais para a avaliação do PAC surgiram, primeiramente, como tradução da língua inglesa, e já foram amplamente estudados e aplicados em diversas populações clínicas, verificando sua validade e eficiência.

O objetivo deste estudo foi verificar a confiabilidade de testes comportamentais para a avaliação do PAC da língua portuguesa do Brasil por meio de teste e reteste em crianças com diagnóstico de transtorno do PAC.

\section{MÉTODOS}

Este estudo foi desenvolvido no Laboratório de Investigação Fonoaudiológica em Processamento Auditivo do Curso de Fonoaudiologia do Departamento de Fisioterapia, Fonoaudiologia e Terapia Ocupacional da Faculdade de Medicina 
da Universidade de São Paulo e foi aprovado pela Comissão de Ética para Análise de Projetos e Pesquisa (CAPPesq) da Diretoria Clínica do Hospital das Clínicas da FMUSP sob o protocolo de pesquisa $\mathrm{n}^{\circ} 731 / 04$.

\section{Casuística}

Foram avaliados 40 indivíduos, sendo 26 do gênero masculino (65\%) e 14 do feminino (35\%), com idades entre sete e 23 anos, com limiares auditivos dentro da normalidade e sem alterações evidentes neurológicas ou psíquicas.

Para esta pesquisa foram utilizados os seguintes materiais: otoscópio da marca Heine®; cabina acústica da marca Siemens ${ }^{\circledR}$ (ANSI S3.1, 1991); analisador de orelha média da marca Grason-Stadler®, modelo GSI-33 (ANSI, 1989); audiômetro da marca Grason-Stadler®, modelo GSI-61 (ANSI S3.6, 1989); fone supra aural modelo TDH-50 (ISSO 7566, 1987); lista de vocábulos para testes de fala ${ }^{(3)}$; Compact Disc Player da marca Sony ${ }^{\circledR}$, com saída direta para o audiômetro; Compact Disc Laser (CD) que contém os testes comportamentais de processamento auditivo(4); sino, agogô, coco e guizo, instrumentos musicais para a realização da avaliação simplificada ${ }^{(4)}$; protocolos para anamnese, registros da avaliação audiológica básica registros dos testes de PAC.

\section{Procedimentos}

Este trabalho foi realizado em duas etapas, sendo que a primeira consistiu na realização da anamnese, da avaliação audiológica básica (meatoscopia, imitanciometria, audiometria tonal e vocal) e da avaliação comportamental do PAC, denominada "teste". Na audiometria tonal, foram considerados normais limiares até 20 dBNA nas frequências de $500 \mathrm{~Hz}$ a $8 \mathrm{kHz}$ (padrão ANSI 69) e indivíduos com limiares tonais superiores foram excluídos do estudo.

$\mathrm{Na}$ segunda etapa do estudo, denominada "reteste", os participantes da pesquisa foram convocados para a realização dos mesmos testes comportamentais do PAC aplicados dentro de um período de uma semana a um mês após o "teste".

Os testes de PAC selecionados e aplicados em cada indivíduo foram de acordo com a idade e as condições de resposta de fala, isto é, na presença de trocas ou distorções articulatórias, foram selecionados testes que não envolvessem respostas verbais.

Todos os testes aplicados pertenciam ao Manual de Avaliação do Processamento Auditivo ${ }^{(4)}$ e, para este estudo, foram selecionados os seguintes testes de processamento auditivo:

Testes da avaliação simplificada: localização da fonte sonora, memória seqüencial para sons verbais e para sons não verbais.

Testes monóticos: Pediatric Speech Intelligibitily Test (PSI) com mensagem competitiva ipsilateral (MCI); Syntetic Sentence Identification (SSI) com mensagem competitiva ipsilateral; Fala com Ruído (FR) ou Identificação de Figuras no Ruído (também denominado PSI com palavras e ruído branco) ${ }^{(4)}$. Nestes testes monóticos a mensagem foi apresentada a 40 dBNS em relação ao valor do Limiar de Reconhecimento de Fala (LRF).
Testes dicóticos: Staggered Spondaic Word Test (SSW); Teste Dicótico de Dígitos (DD); Dicótico Não-Verbal de Escuta Direcionada (DNV). Para os dicóticos, o sinal foi apresentado a 50 dBNS acima do LRF de cada orelha.

Segue um resumo das características e padronizações adotadas para cada um:

- Teste de localização sonora em cinco direções (LOC): Avalia a habilidade de localização da fonte sonora. Pode ser feito com um guizo e o indivíduo é instruído a apontar para a direção de onde acredita provir o som. Espera-se que sejam localizadas pelos menos quatro das cinco posições testadas $^{(5)}$.

- Memória sequencial para sons não verbais com quatro instrumentos (MSNV): Avalia a habilidade de memória sequencial para sons não verbais. Inicialmente, são apresentados os sons de cada instrumento isoladamente, para que o indivíduo possa reconhecê-los e, em seguida, sequências de quatro sons variando a ordem. O indivíduo não deve ver a sequência tocada, mas deve virar-se para apontar e/ou nomear a sequência ouvida. Considera-se bom desempenho a identificação correta de pelo menos duas sequências ${ }^{(5)}$.

- Memória sequencial para sons verbais (MSV): As sílabas $/ \mathrm{pa} /$, /ta/ e /ka/ são apresentadas em três seqüências diferentes para que o indivíduo as repita na ordem em que foram apresentadas. O objetivo do teste é avaliar a memória sequencial para sons verbais. Segundo Pereira, espera-se a identificação correta de pelo menos duas sequências ${ }^{(5)}$.

- PSI: Aplicado sob a forma monótica ipsilateral nas relações sinal/ruído de $0 \mathrm{~dB}$ e $-15 \mathrm{~dB}$. Sendo considerada para esta pesquisa apenas a última relação. Avalia a habilidade de figura-fundo auditiva e associação de estímulos auditivovisuais preferencialmente em crianças pequenas ou indivíduos mais velhos com dificuldades de leitura. Normalidade: $60 \%$ de acertos em ambas as orelhas na forma MCI na relação sinal/ruído de $-15 \mathrm{~dB}^{(6)}$.

- SSI: Aplicado também sob a forma monótica nas relações sinal/ruído de $0 \mathrm{~dB}$ e - $15 \mathrm{~dB}$. Avalia a habilidade de figurafundo auditiva e associação de estímulos auditivo-visuais preferencialmente em jovens ou adultos sem dificuldades de leitura. Normalidade: $60 \%$ de acertos em ambas as orelhas na forma MCI na relação sinal/ruído de $-15 \mathrm{~dB}^{(7)}$. - FR: Aplicado sob a forma monótica na relação sinal/ruído de $+20 \mathrm{~dB}$, considerando-se o audiômetro utilizado e a calibração do ruído tipo White Noise (WN) efetivo. Avalia a habilidade de fechamento auditivo. Não foi aplicado em casos de distúrbio articulatório que comprometesse a compreensão das respostas do sujeito. Normalidade: porcentagens de acertos maiores ou iguais a $68 \%$ na primeira orelha testada e a $70 \%$ na segunda ${ }^{(4)}$.

- Identificação de figuras com ruído ipsilateral ou PSI com palavras e ruído branco: Aplicado na relação sinal/ruído de $+20 \mathrm{~dB}$, sendo a mensagem constituída por monossílabos e o ruído competitivo um ruído do tipo branco. Avalia a habilidade de fechamento auditivo em casos de distúrbio articulatório que comprometa a compreensão das respostas do sujeito ou crianças muito pequenas. Normalidade: porcentagens de acertos maiores ou iguais a 90\% em ambas as orelhas ${ }^{(6)}$. 
- SSW: Teste dicótico que avalia a habilidade figura-fundo para sons linguísticos. Foi utilizado em crianças letradas sem distúrbio articulatório que comprometesse a análise das respostas. Apresenta uma variedade de aspectos quantitativos e qualitativos para análise; entretanto, para medidas estatísticas, apenas as primeiras foram consideradas neste trabalho. A normalidade considerada para este teste foi proposta por Borges ${ }^{(8)}$ e pode ser observada no Quadro 1.

Quadro 1. Padrão de respostas esperadas na adaptação da versão americana do teste SSW para o português do Brasil(9)

\begin{tabular}{|c|c|c|}
\hline Idade (anos) & Direito competitivo & Esquerdo competitivo \\
\hline 7 & $77,5 \%$ & $60,0 \%$ \\
8 & $82,5 \%$ & $75,0 \%$ \\
9 & $85,0 \%$ & $80,0 \%$ \\
10 & $87,5 \%$ & $82,5 \%$ \\
11 & $92,5 \%$ & $85,0 \%$ \\
12 ou mais & $95,0 \%$ & $90,0 \%$ \\
\hline
\end{tabular}

- DD: Teste dicótico que avalia a habilidade figura-fundo para sons linguísticos. Foi utilizado em crianças pequenas e/ou com distúrbio articulatório que comprometesse a análise das respostas. Normalidade esperada para jovens adultos é de $90 \%$ em ambas as orelhas ${ }^{(9)}$.

- DNV: Teste dicótico que avalia a habilidade figura-fundo para sons não linguísticos nas etapas de atenção livre, atenção à orelha direita e esquerda. Para esta análise apenas as etapas de atenção direcionada foram consideradas. Ressalta-se que foram apresentados somente 12 estímulos. Normalidade: 11 acertos para cada orelha, isto é, $91,6 \%{ }^{(10)}$. No reteste, os pacientes foram submetidos novamente à inspeção visual do meato acústico externo (MAE), seguida da triagem timpanométrica e da pesquisa de reflexos acústicos ipsilaterais na frequência de $1 \mathrm{kHz}$. Dessa maneira, excluíramse os pacientes portadores de perdas auditivas condutivas. $\mathrm{O}$ segundo passo foi a confirmação do LRF e posterior aplicação dos mesmos testes de PAC utilizados na primeira avaliação.

Cabe aqui ressaltar que, os dados obtidos em uma avaliação do PAC são de natureza quantitativa e qualitativa, entretanto, neste estudo, apenas os dados quantitativos foram levados em consideração.

Inicialmente, foram computados os acertos e erros em cada teste da bateria de avaliação do PAC e calculada a porcentagem de acerto de cada um. Para a análise estatística do trabalho foram utilizadas medidas da estatística descritiva: média, mediana e desvio padrão, bem como da estatística analítica, por meio da ANOVA, isto é, a análise de variância. Esta última foi utilizada a fim de se estabelecer se há diferença significativa entre os grupos estabelecidos para análise.

O nível de significância deste trabalho foi definido em 0,05 (5\%) e a confiança de $95 \%$.

\section{RESULTADOS}

A seguir serão descritos os resultados obtidos na comparação do desempenho obtido em testes comportamentais de PAC em situação de teste e reteste, realizado após um período máximo de um mês, em 40 indivíduos, sendo $26(65 \%)$ do gênero masculino e 14 (35\%) do feminino, com idades entre sete e 23 anos.

Como na comparação dos acertos obtidos nas orelhas direita e esquerda para cada teste do PAC nas situações teste (T) e reteste (R) não foram encontradas diferenças, os dados foram analisados considerando-se os resultados obtidos em cada orelha, aumentando, assim, o tamanho da amostra.

Na Tabela 1 estão descritas as comparações das médias

Tabela 1. Descrição da análise comparativa de testes comportamentais do PAC em situação de teste e reteste

\begin{tabular}{|c|c|c|c|c|c|c|}
\hline Teste & Situação & Média & Mediana & $\mathrm{DP}$ & Tamanho & Valor de $p$ \\
\hline \multirow[t]{2}{*}{ LOC } & $\mathrm{T}$ & 88,7 & 100 & 14,4 & 40 & 0,084 \\
\hline & $\mathrm{R}$ & 93,5 & 100 & 9,5 & 39 & \\
\hline \multirow[t]{2}{*}{ MSNV } & $\mathrm{T}$ & 63,2 & 66,7 & 35,7 & 40 & 0,113 \\
\hline & $\mathrm{R}$ & 69,2 & 66,7 & 30,6 & 39 & \\
\hline \multirow[t]{2}{*}{ MSV } & $\mathrm{T}$ & 81,2 & 100 & 23,9 & 40 & 1,000 \\
\hline & $\mathrm{R}$ & 78,3 & 100 & 28,8 & 39 & \\
\hline \multirow[t]{2}{*}{ PSI/SSI } & $\mathrm{T}$ & 73,9 & 80,0 & 17,8 & 80 & 0,095 \\
\hline & $\mathrm{R}$ & 78,3 & 80,0 & 15,1 & 80 & \\
\hline \multirow[t]{2}{*}{$\mathrm{FR} / \mathrm{IF}$} & $\mathrm{T}$ & 75,7 & 75,0 & 11,0 & 80 & 0,557 \\
\hline & $\mathrm{R}$ & 74,7 & 76,0 & 11,0 & 80 & \\
\hline \multirow[t]{2}{*}{ DNV } & $\mathrm{T}$ & 82,8 & 91,7 & 21,0 & 78 & 0,451 \\
\hline & $\mathrm{R}$ & 85,3 & 91,7 & 19,7 & 78 & \\
\hline \multirow[t]{2}{*}{ DÍG } & $\mathrm{T}$ & 72,3 & 75,0 & 22,1 & 14 & 0,108 \\
\hline & $\mathrm{R}$ & 81,9 & 85,0 & 11,7 & 14 & \\
\hline \multirow[t]{2}{*}{ SSW } & $\mathrm{T}$ & 73,6 & 72,5 & 17,5 & 64 & 0,258 \\
\hline & $\mathrm{R}$ & 77,0 & 78,8 & 16,4 & 64 & \\
\hline
\end{tabular}

ANOVA $(p \leq 0,05)$

Legenda: $L O C$ = localização sonora; MSNV = memória sequencial para sons não verbais; MSV = memória sequencial para sons verbais; FR = fala com ruído; IF = identificação de figura com ruído; DNV = dicótico não verbal; DIG = dicótico de dígitos; $T$ = situação de teste; $\mathrm{R}=$ situação de reteste; $\mathrm{DP}=$ desvio-padrão 
de acertos nas situações de teste e reteste em todos os testes comportamentais do PAC realizados.

Na Tabela 2 estão descritas as análises comparativas entre as médias de acertos na situação de teste em participantes com resultados dentro da normalidade na avaliação comportamental do PAC.

Na Tabela 3 estão descritas as análises comparativas entre as médias de acertos na situação de reteste em participantes com resultados dentro da normalidade na avaliação comportamental do PAC.
Não foram encontradas diferenças entre teste e reteste nos participantes com resultados de PAC dentro da normalidade.

Não foram encontradas diferenças entre teste e reteste nos participantes com resultados de PAC alterados.

Na Tabela 4 estão descritas as análises comparativas entre participantes com resultados do PAC dentro da normalidade e com resultados alterados na situação de teste.

Na Tabela 5, estão descritas as análises comparativas entre participantes com resultados do PAC dentro da normalidade e com resultados alterados na situação de de reteste.

Tabela 2. Descrição da análise comparativa de testes comportamentais do PAC em situação de teste e reteste em participantes com resultados dentro da normalidade

\begin{tabular}{|c|c|c|c|c|c|c|}
\hline Teste & Situação & Média & Mediana & DP & Tamanho & Valor de $p$ \\
\hline \multirow[t]{2}{*}{ LOC } & $\mathrm{T}$ & 100,0 & 100,0 & 0,0 & 7 & NA \\
\hline & $\mathrm{R}$ & 100,0 & 100,0 & 0,0 & 7 & \\
\hline \multirow[t]{2}{*}{ MSNV } & $\mathrm{T}$ & 90,5 & 100,0 & 16,3 & 7 & 0,611 \\
\hline & $\mathrm{R}$ & 85,7 & 100,0 & 17,8 & 7 & \\
\hline \multirow[t]{2}{*}{ MSV } & $\mathrm{T}$ & 90,5 & 100,0 & 16,3 & 7 & 1,000 \\
\hline & $\mathrm{R}$ & 90,5 & 100,0 & 25,2 & 7 & \\
\hline \multirow[t]{2}{*}{ PSI/SSI } & $\mathrm{T}$ & 87,1 & 90 & 12,7 & 14 & 0,879 \\
\hline & $\mathrm{R}$ & 87,9 & 90 & 11,9 & 14 & \\
\hline \multirow[t]{2}{*}{$\mathrm{FR} / \mathrm{IF}$} & $\mathrm{T}$ & 80,6 & 80,0 & 7,5 & 14 & 0,919 \\
\hline & $\mathrm{R}$ & 80,9 & 80,0 & 7,2 & 14 & \\
\hline \multirow[t]{2}{*}{ DNV } & $\mathrm{T}$ & 98,2 & 100,0 & 3,6 & 14 & 0,297 \\
\hline & $\mathrm{R}$ & 99,4 & 100,0 & 2,2 & 14 & \\
\hline \multirow[t]{2}{*}{ SSW } & $\mathrm{T}$ & 95,2 & 97,5 & 5,5 & 14 & 0,401 \\
\hline & $\mathrm{R}$ & 91,1 & 95,0 & 17,1 & 14 & \\
\hline
\end{tabular}

ANOVA $(p \leq 0,05)$

Legenda: $L O C$ = localização sonora; MSNV = memória sequencial para sons não verbais; MSV = memória sequencial para sons verbais; FR = fala com ruído; IF = identificação de figura com ruído; DNV = dicótico não verbal; DIG = dicótico de dígitos; $T$ = situação de teste; $\mathrm{R}=$ situação de reteste; $\mathrm{DP}=$ desvio-padrão

Tabela 3. Descrição da análise comparativa de testes comportamentais do PAC em situação de teste e reteste em participantes com resultados alterados

\begin{tabular}{|c|c|c|c|c|c|c|}
\hline Teste & Situação & Média & Mediana & DP & Tamanho & Valor de $p$ \\
\hline \multirow[t]{2}{*}{ LOC } & $\mathrm{T}$ & 86,3 & 80 & 14,8 & 33 & 0,064 \\
\hline & $\mathrm{R}$ & 92,1 & 100 & 9,9 & 32 & \\
\hline \multirow[t]{2}{*}{ MSNV } & $\mathrm{T}$ & 57,3 & 66,7 & 36,2 & 33 & 0,325 \\
\hline & $\mathrm{R}$ & 65,7 & 66,7 & 31,7 & 32 & \\
\hline \multirow[t]{2}{*}{ MSV } & $\mathrm{T}$ & 79,2 & 100 & 25,0 & 33 & 0,616 \\
\hline & $\mathrm{R}$ & 75,8 & 66,7 & 29,2 & 32 & \\
\hline \multirow[t]{2}{*}{ PSI/SSI } & $\mathrm{T}$ & 71,1 & 70,0 & 17,5 & 66 & 0,071 \\
\hline & $\mathrm{R}$ & 76,2 & 80,0 & 15,0 & 66 & \\
\hline \multirow[t]{2}{*}{$\mathrm{FR} / \mathrm{IF}$} & $\mathrm{T}$ & 74,6 & 72,0 & 11,4 & 66 & 0,510 \\
\hline & $\mathrm{R}$ & 73,3 & 72,0 & 11,3 & 66 & \\
\hline \multirow[t]{2}{*}{ DNV } & $\mathrm{T}$ & 79,4 & 91,7 & 21,7 & 64 & 0,464 \\
\hline & $\mathrm{R}$ & 82,2 & 91,7 & 20,5 & 64 & \\
\hline \multirow[t]{2}{*}{ DÍG } & $\mathrm{T}$ & 72,3 & 75,0 & 22,1 & 14 & 0,160 \\
\hline & $\mathrm{R}$ & 81,9 & 85,0 & 11,7 & 14 & \\
\hline \multirow[t]{2}{*}{ SSW } & $\mathrm{T}$ & 67,6 & 70,0 & 14,7 & 50 & 0,058 \\
\hline & $\mathrm{R}$ & 73,1 & 75,0 & 14,0 & 50 & \\
\hline
\end{tabular}


Tabela 4. Descrição da análise comparativa entre médias de acertos de participantes com resultados de PAC dentro da normalidade e com resultados alterados na situação de teste

\begin{tabular}{|c|c|c|c|c|c|c|}
\hline Teste & Situação & Média & Mediana & DP & Tamanho & Valor de $p$ \\
\hline \multirow[t]{2}{*}{ LOC } & $\mathrm{N}$ & 100,0 & 100,0 & NA & 7 & $0,020^{*}$ \\
\hline & $A$ & 86,0 & 80,0 & 15,0 & 32 & \\
\hline \multirow[t]{2}{*}{ MSNV } & $\mathrm{N}$ & 90,5 & 100,0 & 16,3 & 7 & $0,024^{*}$ \\
\hline & $A$ & 57,3 & 67,0 & 36,2 & 32 & \\
\hline \multirow[t]{2}{*}{ MSV } & $\mathrm{N}$ & 90,5 & 100,0 & 16,3 & 7 & 0,263 \\
\hline & $A$ & 79,2 & 100 & 25,0 & 32 & \\
\hline \multirow[t]{2}{*}{ PSI/SSI } & $\mathrm{N}$ & 87,1 & 90,0 & 12,7 & 14 & $0,002^{*}$ \\
\hline & $A$ & 71,1 & 70,0 & 17,5 & 66 & \\
\hline \multirow[t]{2}{*}{$\mathrm{FR} / \mathrm{IF}$} & $\mathrm{N}$ & 80,6 & 80,0 & 7,5 & 14 & 0,066 \\
\hline & $A$ & 74,6 & 72,0 & 11,4 & 66 & \\
\hline \multirow[t]{2}{*}{ DNV } & $\mathrm{N}$ & 98,2 & 100,0 & 3,6 & 14 & $0,002^{*}$ \\
\hline & A & 79,4 & 91,7 & 21,7 & 64 & \\
\hline \multirow[t]{2}{*}{ SSW } & $\mathrm{N}$ & 95,2 & 97,5 & 5,5 & 14 & $<0,001^{*}$ \\
\hline & $A$ & 67,6 & 70,0 & 14,7 & 50 & \\
\hline
\end{tabular}

*Valores significativos $(\mathrm{p} \leq 0,05)$ - ANOVA

Legenda: $L O C=$ localização sonora; $M S N V=$ memória sequencial para sons não verbais; $M S V=$ memória sequencial para sons verbais; FR = fala com ruído; IF = identificação de figura com ruído; $\mathrm{DNV}=$ dicótico não verbal; $\mathrm{N}$ = resultados do PAC dentro da normalidade; $\mathrm{A}$ = resultados do PAC alterados; $\mathrm{DP}=$ desvio-padrão

Tabela 5. Descrição da análise comparativa entre médias de acertos de participantes com resultados de PAC dentro da normalidade e com resultados alterados na situação de reteste

\begin{tabular}{|c|c|c|c|c|c|c|}
\hline Teste & Situação & Média & Mediana & DP & Tamanho & Valor de $p$ \\
\hline \multirow[t]{2}{*}{ LOC } & $\mathrm{N}$ & 100,0 & 100 & 0 & 7 & $0,044^{*}$ \\
\hline & $A$ & 92,1 & 100 & 10,0 & 33 & \\
\hline \multirow[t]{2}{*}{ MSNV } & $\mathrm{N}$ & 86,0 & 100 & 17,8 & 7 & 0,116 \\
\hline & A & 65,7 & 66,7 & 32,0 & 33 & \\
\hline \multirow[t]{2}{*}{ MSV } & $\mathrm{N}$ & 90,5 & 100 & 25,0 & 7 & 0,224 \\
\hline & A & 75,8 & 66,7 & 29,2 & 33 & \\
\hline \multirow[t]{2}{*}{ PSI/SSI } & $\mathrm{N}$ & 87,9 & 90,0 & 11,9 & 14 & $0,008^{*}$ \\
\hline & A & 76,2 & 80,0 & 15,0 & 66 & \\
\hline \multirow[t]{2}{*}{$\mathrm{FR} / \mathrm{IF}$} & $\mathrm{N}$ & 80,9 & 80,0 & 7,2 & 14 & $0,020^{*}$ \\
\hline & A & 73,3 & 92,0 & 11,3 & 66 & \\
\hline \multirow[t]{2}{*}{ DNV } & $\mathrm{N}$ & 99,4 & 100 & 2,2 & 14 & $0,003^{*}$ \\
\hline & A & 82,2 & 91,7 & 20,5 & 64 & \\
\hline \multirow[t]{2}{*}{ SSW } & $\mathrm{N}$ & 91,1 & 95,0 & 17,1 & 14 & $<0,001^{\star}$ \\
\hline & A & 73,1 & 75,0 & 14,0 & 50 & \\
\hline
\end{tabular}

*Valores significativos $(p \leq 0,05)$ - ANOVA

Legenda: $L O C=$ localização sonora; MSNV = memória sequencial para sons não verbais; MSV = memória sequencial para sons verbais; FR = fala com ruído; IF = identificação de figura com ruído; DNV = dicótico não verbal; $\mathrm{N}=$ resultados do PAC dentro da normalidade; $\mathrm{A}=$ resultados do PAC alterados; $\mathrm{DP}=$ desvio-padrão

Foram encontradas diferenças para os testes de LS, MSNV, DNV e SSW na comparação entre participantes com resultados normais e alterados para o PAC na situação de teste.

\section{DISCUSSÃO}

A confiabilidade na avaliação comportamental do PAC tem como fundamento a comprovação da estabilidade dos testes aplicados, verificada por meio de situações de teste e de reteste.

De acordo com os resultados descritos na Tabela 1, verificou-se melhora de desempenho nos testes comportamentais do PAC na situação de reteste para todos os testes exceto para o MSV e para o FR/Identificação de Figuras no Ruído. No entanto, não foram encontradas diferenças para nenhum teste na comparação com o respectivo reteste.

A melhora no desempenho da segunda avaliação realizada foi observada por diversos autores, os quais justificaram este achado pelo efeito da familiaridade com a situação do teste ou pelo efeito de aprendizagem ${ }^{(11,12)}$.

Apesar da melhora de desempenho verificada em alguns testes neste estudo, esta não foi significativa, não alterando, assim, o resultado encontrado. Sendo assim, de maneira geral, 
estes achados estiveram de acordo com diversos autores, que encontraram coeficiente de estabilidade de 0,82 com relação ao teste PSI $^{(13)}$, e que encontraram de 0,77 com relação ao teste de Dicótico de Dígitos ${ }^{(14)}$. No entanto, alguns autores referiram não encontrar boa correlação entre o teste e reteste na bateria de testes do PAC denominada SCAN, e atribuíram isso ao grande intervalo de tempo ocorrido entre as situações, aproximadamente de seis meses ${ }^{(15)}$. Do mesmo modo, autores que realizaram o reteste do teste Fala Filtrada verificaram melhora significante na resposta tanto em crianças como em $\operatorname{adultos}^{(16,17)}$.

Com relação à análise realizada em participantes com resultados dentro da normalidade, como descritos na Tabela 2, verificou-se um desempenho bastante homogêneo neste grupo ao se verificar os valores de média, mediana e desvio padrão. Da mesma maneira, não foram encontradas diferenças estatisticamente significantes entre as situações de teste e reteste. Além disso, dos sete testes analisados, quatro apresentaram a mesma média de respostas no reteste. Isto pode indicar que, na prática clínica, os resultados de testes de PAC encontrados dentro da normalidade possuem boa confiabilidade.

Quanto aos participantes com resultados alterados para o PAC, as respostas encontradas foram mais variáveis, com desvios padrões maiores, e em seis testes verificou-se melhora de respostas na situação reteste. No entanto, do mesmo modo, estas diferenças não foram estatisticamente significantes (Tabela 3).

$\mathrm{Na}$ comparação entre os dois grupos, com resultados do PAC normais e alterados, na situação de teste, descritos na Tabela 4, verificou-se diferença estatisticamente significante em todos os testes, com exceção do teste Fala com Ruído, onde houve apenas uma tendência já que o p-valor foi de 0,066.

Com relação à situação de reteste, também houve diferenças na maioria dos testes entre os dois grupos, com exceção dos testes de Memória Sequencial para Sons Verbais e Não Verbais (Tabela 5). Observou-se, no entanto, que na situação de reteste o grupo com alteração de PAC apresentou melhor desempenho nos testes, o contrário do verificado no grupo sem alteração de PAC. Estes dados não estiveram de acordo com outros estudos, pois estes autores não verificaram diferenças no desempenho entre crianças com e sem alteração de PAC na situação de reteste ${ }^{(12,16)}$.

Neste estudo, não foram encontradas entre a situação de teste e reteste tanto para o grupo de participantes sem alteração de PAC como para o grupo com alteração de PAC.

No entanto, a melhora de desempenho na situação de reteste foi verificada em ambos os grupos, embora tenha sido maior no grupo com alteração de PAC, refletindo o efeito de familiaridade ou aprendizagem do teste.

Acredita-se que, além da dificuldade na realização dos próprios testes, indivíduos com alteração de PAC são mais suscetíveis à influência de outros fatores, como motivação, insegurança, desconhecimento dos testes, atenção, memória, aprendizagem, entre outros, ocasionando maior variação nos resultados encontrados nas situações de teste e reteste.

No entanto, cabe ressaltar que mesmo sendo verificadas estas variações, os resultados encontrados como normais e alterados nas avaliações de teste e reteste foram constantes entre as duas situações para os dois grupos estudados. Este fato reforça a confiabilidade na aplicação clínica dos testes de PAC analisados neste estudo.

\section{CONCLUSÃO}

Este estudo analisou a estabilidade de resultados de testes comportamentais de PAC, verificada por meio das situações de teste e reteste.

Neste estudo, foi possível concluir que os testes comportamentais analisados foram confiáveis, pois não foram encontradas na comparação entre a situação de teste e reteste.

Com relação à análise dos grupos de participantes com resultados normais e com resultados alterados de PAC, também não foram encontradas entre a situação de teste e reteste. No entanto, verificou-se maior variabilidade de respostas na situação de reteste no grupo de participantes com alteração de PAC.

\begin{abstract}
Purpose: To verify the reliability of a few Central Auditory Processing (CAP) tests through a test-retest study. Methods: Forty Portuguese speaking volunteer subjects were randomly selected and evaluated on CAP abilities in the situations of test and retest, with interval periods that varied from one week to one month. The following tests were applied: localization, verbal and non-verbal sequential memory, two monotic and two dichotic tests. These last ones were selected according to each subject's age and response conditions. Results: There was no difference between the tested ears, nor between the test and retest situations for the entire group. When the performances of subjects indicating an auditory processing disorder were compared to those whose results were within normal limits there was a variation in the majority of the tests, in both situations. Conclusion: The CAP tests used in this study indicated their reliability through the test-retest.
\end{abstract}

Keywords: Hearing; Hearing disorders; Auditory perception; Auditory perceptual disorders; Hearing tests 


\section{REFERÊNCIAS}

1. Jerger J, Musiek F. Report of the Consensus Conference on the Diagnosis of Auditory Processing Disorders in School-Aged Children. J Am Acad Audiol. 2000;11(9):467-74.

2. Bocca E, Calearo C, Cassinari V. A new method for testing hearing in temporal lobe tumours; preliminary report. Acta Otolaryngol. 1954;44(3):219-21.

3. Russo ICP, Santos TMM. Logoaudiometria. In: Santos TMM, Russo ICP. A prática da audiologia clínica. São Paulo: Cortez; 1986. p. 81-98.

4. Pereira LD, Schochat E. Processamento auditivo central: manual de avaliação. São Paulo: Lovise; 1997.

5. Pereira LD. Processamento auditivo. Temas Desenvolv. 1993;2(11):7-14.

6. Almeida CIR, Campos MI, Almeida RR. Logoaudiometria pediátrica (PSI). Rev Bras Otorrinolaringol. 1988;54(3):73-6.

7. Aquino AMCM, Almeida CIR, Oliveira JAA. Teste de identificação de sentenças sintéticas (SSI) em português com mensagem competitiva: uma padronização. Rev Bras Otorrinolaringol.1993;59(3):160-3.

8. Borges ACC. Adaptação do teste SSW para a língua portuguesa. Nota preliminar. Acta AWHO. 1986;5(Supl 1):38-40.

9. Santos MFC, Pereira LD. Escuta com dígitos. In: Pereira LD, Schochat E. Processamento auditivo central: manual de avaliação. São Paulo: Lovise; 1997. p. 147-50.

10. Ortiz KZ, Pereira LD. Teste dicótico não verbal de escuta direcionada. In: Pereira LD, Schochat E. Processamento auditivo central: manual de avaliação. São Paulo: Lovise; 1997.
11. Wilson RH, Bell TS, Koslowski JA. Learning effects associated with repeated word-recognition measures using sentence materials. J Rehabil Res Dev. 2003;40(4):329-36.

12. Heath SM, Hogben JH. The reliability and validity of tasks measuring perception of rapid sequences in children with dyslexia. J Child Psychol Psychiatry. 2004;45(7):1275-87.

13. Jerger S. Evaluation of central auditory function in children. In: Keith RW, editor. Central auditory and language disorders in children. San Diego: College-Hill Press; 1982. p. 30-60.

14. Musiek FE, Gollegly KM, Kibbe KS, Verkest-Lenz SB. Proposed screening test for central auditory disorders: follow-up on the dichotic digits test. Am J Otol. 1991;12(2):109-13.

15. Keith RW. Development and standardization of SCAN-C Test for Auditory Processing Disorders in Children. J Am Acad Audiol. 2000;11(8):438-45.

16. Amos NE, Humes LE. SCAN test-retest reliability for first- and thirdgrade children. J Speech Lang Hear Res. 1998;41(4):834-45.

17. Neijenhuis KA, Stollman MH, Snik AF, Van der Broek P. Development of a central auditory test battery for adults. Audiology. 2001;40(2):6977. 